die bei, der Monosubstitution rein hervortritt zu unterscheiden von einem Symmetrieeffekt b), der rom elektrochemischen Charakter der Substituenten gar nicht, dagegen um so mehr von dessen Masse abhängt. Bernoulli und $W_{e g e}{ }^{1}$ ) haben diesen ron ihnen an der Diallylmalonsäure zuerst aufgefundenen Effekt b) als wachsende dynamische Stabilisierung des Molekelkreisels gedeutet. Der Befund an den beiden Dihalogen-malonsäuren bestätigt diese Auffassung aufs Beste.

Basel, Physikalisth-Chemische Anstalt der Universität.

\title{
Die Herstellung von Nitroanisol aus Nitrochlorbenzol von
}

\author{
A. V. Blom.
}

(3. XI. 21.)

Erhitzt man o- oder p-Nitrochlorbenzol bei Gegenwart von freiem 1lkali mit Methylalkohol, so wird das Chloratom gegen die Methoxylgruppe ausgetauscht. Die Reaktion verläuft wie die Phenetolbildung, über deren Mechanismus wir früher ${ }^{2}$ ) berichtet haben. I)ie dort geschillerte Untersuchungsmethode lässt sich auf dic Anisolbildung ohne weiteres übertragen. Für alle Einzelheiten der Methodik und der Bezeichnungsweise wird auf jene Publikation verwiesen.

Eine Reihe von Vorversuchen liess erkennen, dass die Reduktionswirkung des Methylalkoholates auf die Nitrogruppe sich erst oberhalb $70^{\circ}$ entfaltet, wie wir das bereits beim Äthylalkoholat festgestellt haben. Arbeitet man also unter gewöhnlichem I.ruck, so kann die Bildung von Azoxyverbindungen rernachlässigt werden, selbst wenn man das Reaktionsgemisch his zum Sieden erhitzt.

1, 1. c. 530.

$\Rightarrow$ Helv. 4, 297 (1921). 


\section{Experimentelle Daten.}

$15,75 \mathrm{gr}$ Nitrochlorbenzol wurden in $1.5,5 \mathrm{~cm}^{3}$ warmem Methylalkohol, 5,62 gr reines Kaliumhydroxyd in $44,5 \mathrm{~cm}^{3}$ Wasser gelöst. Nach dem Vermischen beider Lösungen lies: man rückfliessend kochen.

Totalyolumen $200 \mathrm{~cm}^{3}$,
molare Konz. $0,50$.
Titersumme $\quad 25,0 \mathrm{~cm}^{3}$

Von Zeit zu Zeit wurden $5 \mathrm{~cm}^{3}$-Proben heiss gezogen und sofort mit kaltem Wasser verdünnt. Die Titration mit 0,1-n. Salpetersäure unter Verwendung von Phenolphtalë̈n-, Brillantgell- resp. Kongopapier ergab die 'Titer: $\mathbf{P h}, \mathbf{B r}$ resp. Ko. Dic genau neutrali-ierte Iösungr diente, nach Zusatz von etwas Kaliumchromatlösung. \%ur Feststellung des Chlorgehaltes nach Mohr (Ag-titer).

Der Ph-titer wurde mit der Korrektion-zahl as virehen, die wir in einer früheren Abhandlung abgeleitet hahen ${ }^{1}$ ).

Jer $\mathrm{Ag}$-titer ist von der Äther- und Phenolbildung allein abhängig. Der Ko-titer wird durch die Äther- und Azoxybildung beeinflusst. Der Ph-titer gibt ein Mass für die Summe aller chrei Reaktionen. Kamn man die Reduktionswirkung des Alkoholates vernathliissigen, so jis die Differenz der beiden Alkalinitätstiter proportional der Phenolkonzentration. Wir haben früher ${ }^{2}$ die empiriche formel anfoestellt:

$$
4,3\left[\mathrm{Ko}-\left(\mathrm{Ph}_{\mathrm{H}}+2\right)\right]=\% \text { Nitrophenol. }
$$

Versuch 1. p-Nitrochlorbenzol.

\begin{tabular}{|c|c|c|c|c|c|c|c|c|}
\hline \multirow[b]{2}{*}{$\mathrm{t}$} & \multicolumn{3}{|c|}{0,1 -n. $\mathrm{HNO}_{3} \mathrm{~cm}^{3}$} & \multirow{2}{*}{$\begin{array}{c}\mathrm{AgNO}_{3} \\
\mathrm{~cm}^{3}\end{array}$} & \multirow{2}{*}{$\begin{array}{l}\text { Nitrophenol } \\
\mathrm{Ko}-(\mathrm{Ph}+2)\end{array}$} & \multirow{2}{*}{$\begin{array}{c}\text { Azoxy } \\
A g+P h+2\end{array}$} & \multicolumn{2}{|c|}{ Prozentgehalt } \\
\hline & $\mathrm{Ph}$ & $\mathrm{Br}$ & Ko & & & & $\begin{array}{l}\mathrm{KCl} \\
(\mathrm{Ag})\end{array}$ & $\begin{array}{l}\mathrm{KOH} \\
(\mathrm{Ko})\end{array}$ \\
\hline 0 & 22,9 & 23,6 & 24,9 & 0,3 & - & - & - & - \\
\hline 2 & - & 16,0 & 16,9 & 8,3 & - & - & 33 & 68 \\
\hline 6 & 8,1 & 8,7 & 9,1 & 14,9 & 0 & 25,0 & 60 & 36 \\
\hline 9 & 6,4 & 6,9 & 8,1 & 17,3 & 0 & 25,7 & 69 & 32 \\
\hline 23 & 2,9 & 3,5 & $4, \tilde{\tilde{2}}$ & 21,0 & 0,4 & 25,9 & 84 & 18 \\
\hline 48 & 1,4 & 1,9 & 3,7 & 22,2 & 0,5 & 25,6 & $8 x$ & 15 \\
\hline
\end{tabular}

Ausbeute: p-Nitroanisol $86 \%+$ p-Nitrophenol $2 \%$; kein Dichlorazoxybenzol. Kontrolltitrierung der Endlauge: $93 \% \mathrm{KCl}$.

Anfängliche Titersumme: $(\mathrm{Ko}+\mathrm{Ag})=25,2$ (statt 25).

Nitrophenol berechnet: $4,3[\mathrm{Ko}-(\mathrm{Ph}+2)]=2,1 \%$.
1) Helv. 4, 512 (1921).
2) 1. c. 513 . 
Versuche 2 und 3.

o-Nitrochlorbenzol.

\begin{tabular}{|c|c|c|c|c|c|c|c|c|}
\hline \multirow[b]{2}{*}{$t$} & \multicolumn{3}{|c|}{$0,1-n . \mathrm{HNO}_{3} \mathrm{~cm}^{3}$} & \multirow{2}{*}{$\begin{array}{c}\mathrm{AgNO}_{3} \\
\mathrm{~cm}^{3}\end{array}$} & \multirow{2}{*}{$\begin{array}{l}\text { Nitrophenol } \\
\mathrm{Ko}-(\mathrm{Ph}+2)\end{array}$} & \multirow{2}{*}{$\begin{array}{c}\text { Azoxy } \\
\mathrm{Ag}+\mathrm{Ph}+2\end{array}$} & \multicolumn{2}{|c|}{ Prozentgehalt } \\
\hline & $\mathrm{Ph}$ & $\mathrm{Br}$ & Ko & & & & $\begin{array}{l}\mathrm{KCl} \\
(\mathrm{Ag})\end{array}$ & $\begin{array}{r}\mathrm{KOH} \\
\text { (Ko) }\end{array}$ \\
\hline 0 & 23,4 & 24,2 & 25,1 & 0,2 & - & - & - & - \\
\hline 3 & 19,6 & 19,9 & 20,7 & 4,6 & - & 26,2 & 19 & 83 \\
\hline 8 & 13,7 & 15,3 & 16,0 & 9,4 & 0,3 & 25,1 & 38 & 64 \\
\hline 23 & 7,1 & 7,8 & 9,3 & 14,8 & 0,2 & 23,9 & 59 & 37 \\
\hline 32 & 5,5 & 6,7 & 8,4 & - & 0,9 & - & - & 33 \\
\hline 51 & 3,6 & 4,6 & 6,8 & 18,8 & 1,2 & 24,4 & 75 & 27 \\
\hline 95 & 0,9 & 2,2 & 5,1 & 20,0 & 2,2 & $22,9 ?$ & 80 & 20 \\
\hline
\end{tabular}

Ausbeute: o-Nitroanisol $84 \%+$ o-Nitrophenol $3,6 \%$; kein Dichlorazoxybenzol. Kontrolltitrierung der Endlauge $86,4 \% \mathrm{KCl}$.

Anfängliche Titersumme: $(\mathrm{Ko}+\mathrm{Ag})=25,3$ (statt 25).

\begin{tabular}{|c|c|c|c|c|c|c|c|}
\hline \multirow[b]{2}{*}{$\mathbf{t}$} & \multicolumn{2}{|c|}{$0,1-\mathrm{n} . \mathrm{HNO}_{3} \mathrm{~cm}^{3}$} & \multirow{2}{*}{$\begin{array}{c}\mathrm{AgNO}_{3} \\
\mathrm{~cm}^{3}\end{array}$} & \multirow{2}{*}{$\begin{array}{l}\text { Nitrophenol } \\
\mathrm{Ko}-(\mathrm{Ph}+2)\end{array}$} & \multirow{2}{*}{$\begin{array}{c}\text { Azoxy } \\
\mathrm{Ag}+\mathrm{Ph}+2\end{array}$} & \multicolumn{2}{|c|}{ Prozentgehalt } \\
\hline & $\mathrm{Ph}$ & Ko & & & & $\begin{array}{l}\mathrm{KCl} \\
(\mathrm{Ag})\end{array}$ & $\begin{array}{r}\mathbf{K O H} \\
(\mathbf{K o})\end{array}$ \\
\hline 0 & 23,8 & 26,1 & 0,4 & 0,3 & - & -- & - \\
\hline 4 & 16,3 & 19,7 & 7,2 & 1,4 & 25,5 & 29 & 79 \\
\hline 22 & 7,0 & 10,2 & 16,1 & 1,2 & 25,1 & 64 & 41 \\
\hline 52 & 3,8 & 6,8 & 19,2 & 1,0 & 25,0 & 77 & 27 \\
\hline 75 & 2,0 & 5,9 & 21,1 . & 1,9 & 25,1 & 84 & 24 \\
\hline 99 & 1,5 & 5,5 & $21,9^{\circ}$ & 2,0 & 25,4 & 87 & 22 \\
\hline
\end{tabular}

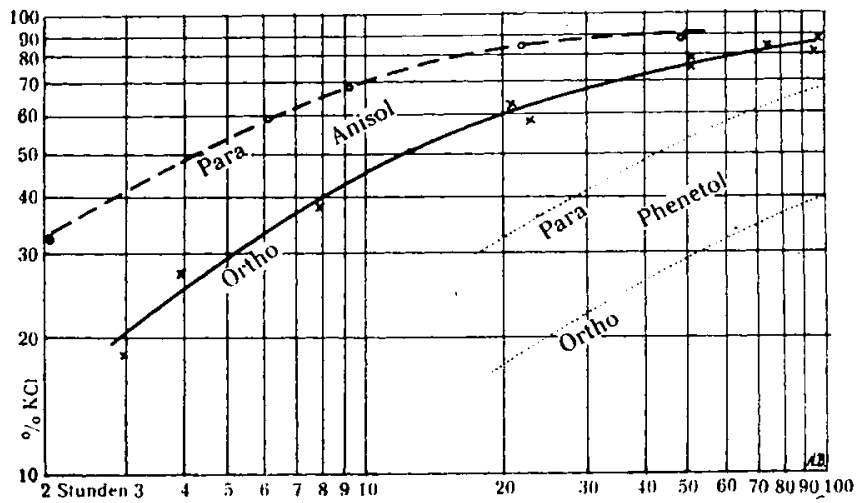

Fig. 1. 
Die Messungsresultate lassen sich wiederum am besten in einem logarithmischen Koordinatensystem graphisch darstellen. Fig. 1 zeigt die Zunahme von Chlorionen im Reaktionsgemisch; die Abszissen entsprechen der Summe von jeweilen vorhandenem Nitroanisol und Nitrophenol. Die Hälfte des angewandten p-Nitrochlorbenzols ist bereits nach vier Stunden umgesetzt, während die Orthoverbindung etwa 13 Stunden braucht. Die Äthylierung bei $70^{\circ}$ unter ähnlichen Konzentrationsverhältnissen erfordert 44 resp. 155 Stunden.

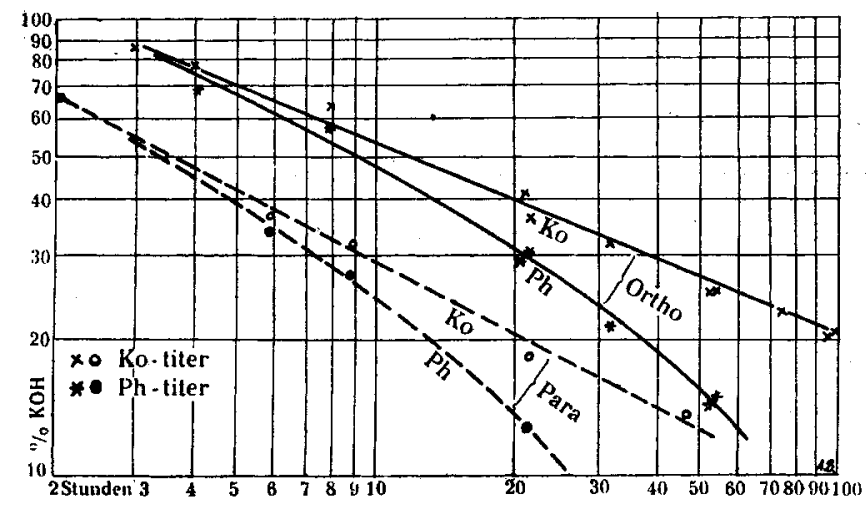

Fig. 2.

Fig. 2 stellt die Abnahme der Alkalinitätstiter dar. Der von je zwei zusammengehörigen Ko- und $\mathrm{Ph}$-Kurven eingeschlossene Bezirk ist ein Mass für das gebildete Nitrophenol. Im logarithmischen Schaubild sind diese beiden Areale einander kongruent, woraus man schliessen darf, dass beide isomeren Nitrophenole demselben Bildungsgesetz unterliegen. Die Ausbeute an diesem Nebenprodukt scheint vorzugsweise eine Zeitfunktion zu sein. Da bei der Paraverbindung das Gleichgewicht rascher erreicht wird (bei ca. 30 Stunden, gegen 100 Stunden beim Orthoderivat), so muss die Phenolbildung früher zum Stehen kommen. Der sterische Einfluss der Nitrogruppe ist für diese Nebenreaktion von geringer Bedeutung.

Aus den drei mitgeteilten Versuchen ergibt sich eine Bestätigung der früher ${ }^{1}$ ) berechneten Korrektionszahl für den $\mathrm{Ph}$-titer. Die Nulltiterdifferenzen $\left(\mathrm{Ko}_{\mathrm{O}}-\mathrm{Ph}\right)$ betragen:



1) Helv. 4, 513 (1921). 
Beim Versuch 2 sollte nach dem Verlauf der Titerkurven eine geringfügige Bildung von Dichlorazoxybenzol stattgefunden haben (rinken der Titersumme). Beim Parallelversuch 3 sind die Titersummen dagegen konstant. Wir haben dieselbe Erscheinung bei den Ïthylierungsversuchen auch häufig beobachtet, ohne eine sichere Erklärung dafür geben zu können.

Kinetik der Reaktionen.

Aus Fig. 1 können wir die ausgeglichenen Werte für gebildetes Kaliumchloril ablesen; wir setzen die daraus berechneten Reaktionskonstanten II. und III. Ordnung daneben:

\begin{tabular}{|r|c|c|c|c|c|}
\hline \multirow{2}{*}{$t$} & \multicolumn{2}{|c|}{ Paranitrochlorbenzol } & \multicolumn{3}{c|}{ Orthonitrochlorbenzol } \\
\cline { 2 - 6 } & $\% \mathrm{KCl}$ & $10^{4} \cdot \mathrm{k}_{2}$ & $\% \mathrm{KCl}$ & $10^{4} \cdot \mathrm{k}_{2}$ & $10^{4} \cdot \mathrm{k}_{3}$ \\
\hline \hline \multirow{2}{*}{5} & 54 & 23 & 29 & 8,2 & 0,10 \\
10 & 70 & 23 & 45 & 8,2 & 0,11 \\
15 & 77 & 22 & 53 & 7,5 & 0,12 \\
20 & 83 & 24 & 60 & 7,5 & 0,15 \\
50 & 91 & 20 & 76. & 6,3 & 0,16 \\
100 & 92 & 12 & 86 & 6,1 & 0,25 \\
\hline
\end{tabular}

Beim p-Nitrochlorbenzol findet somit eine Reaktion Il. Ordnung statt $\left(\mathrm{k}_{2}=23 \cdot 10^{-4}\right)$, bis nach 20 Stunden über $80 \%$ umgesetzt sind. Die Orthoverbindung reagiert nicht nach einer bestimmten Ordnung. Wahrscheinlich ist die stärkere Phenolbildung schuld an der Anomalie.

Die entsprechenden Konstanten für die Phenetole findet man auf $\$$. 317 dieses Jahrganges. Dort hat sich in der Tabelle ein Druckfehler eingeschlichen. In der vorletzten Spalte der Tabelle muss die Überschrift heissen: $10^{4} \cdot k_{3}$ statt $10^{4} \cdot k_{2}$.

Nach der Formel

$$
\mathrm{X}=\frac{10^{4} \cdot \mathrm{k}_{2} \cdot \mathrm{t}}{\mathrm{l}+100 \cdot \mathrm{k}_{2} \cdot \mathrm{t}} \% \mathrm{~K}(\mathrm{l}
$$

kann man beim p-Nitrochlorbenzol die Timsetzung für jeden beliehigen Zeitpunkt vorausberechnen. Für den Versuch 1 erhalten wir folgende Zusammenstellung:

\begin{tabular}{|c|c|c|}
\hline \multirow{2}{*}{$\mathrm{t}$} & \multicolumn{2}{|c|}{ Emsetzung \% } \\
\cline { 2 - 3 } & berechnet & gefunden \\
\hline \hline 6 & 58 & 60 \\
9 & 67 & 69 \\
23 & 84 & 84 \\
48 & 92 & 88 \\
\hline
\end{tabular}


Die Übereinstimmung ist gut, wenn man die Vursuchsfehlergrenzen berücksichtigt.

Für die Orthoverbindung lässt sich leirler kein so einfaches mathematisches Modell des Vorganges aufstellen; man muss schon seine Zuflucht zur graphischen Interpolation nehmen.

\section{Schluss.}

Die Cmsetzung der beiden reaktionsfähigen Nitrochlorbenzol. mit Alkoholaten ist studiert worden. Die Konp̧wenten waren

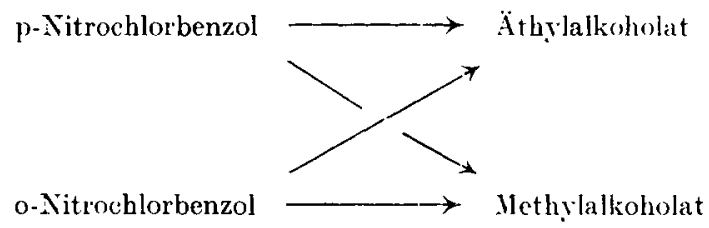

Die Methodik der Untersuchung bestand in ciner Kombination ron verschiedenen Titrationen. Es gelang, die Bildungkgeschwindigkeit jedes einzelnen Reaktionsteilnehmers mesend zu rerfolgen.

Interhalb $70^{\circ}$ ist die Reduktionswirkung intermediär quelildeten Alkoholates gering; sie kann praktisch rernachlässigt werden. Die Zustandsdiagramme können dann durch dir F̈ig. 3 und 4 wiedergegeben werden. Sie gelten für die molare Konzentration 0,50 .

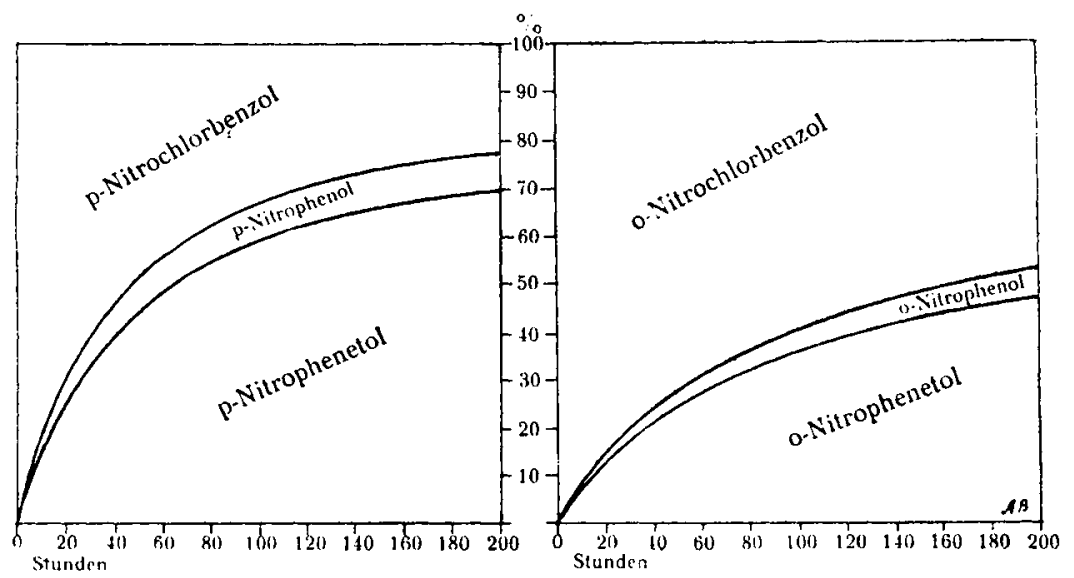

Fig. 3. 


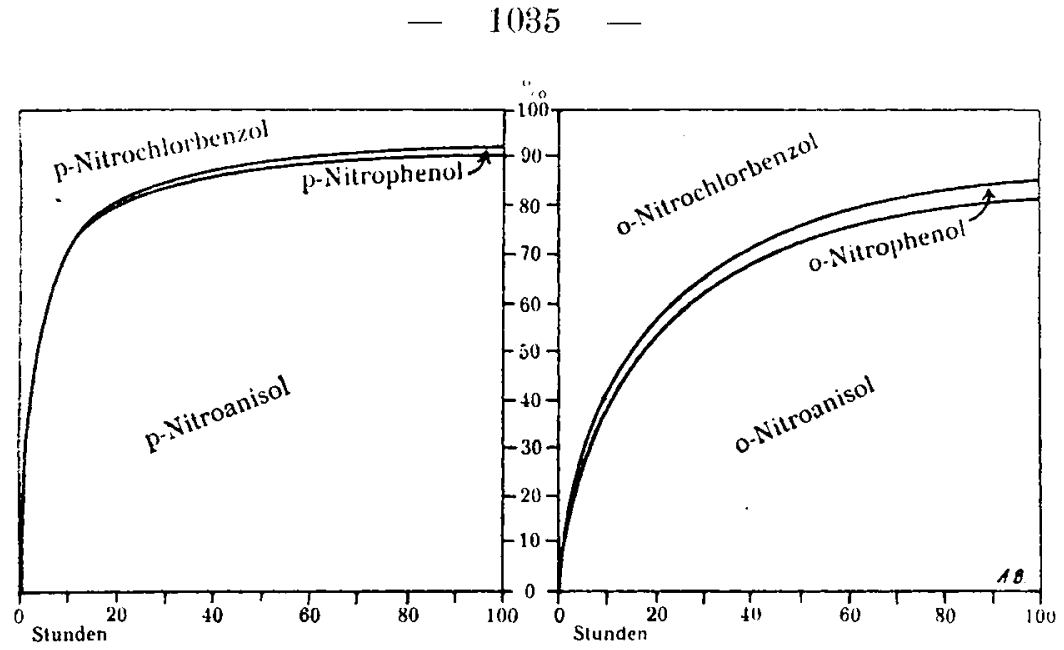

Fig. 4.

Die Ausbeuten an Nitrophenoläther und die Emsetzungsgeschwinligkeiten sind nicht bloss von Temperatur und Zeit, sondern auch von ler Menge und der Zusammensetzung des Lösungsmediums abhängig. Beschleunigende Faktoren vergrössern die Ausbeute an Äther, sofern sie nicht gleichzeitig die Nebenreaktionen auch fördern. Günstig wirken in diesem Sinne hohe Alkoholkonzentration und ein Minimum an I.̈̈sungsmittel. Temperaturerhöhung über $70^{\circ}$ und grosser Alkaliüberschuss wirken wegen der vermehrten Bildung von Dichlorazoxybenzol resp. Nitrophenol eher schädigend.

Die Verseifung bereits gebildeten $̈$ thers kommt praktisch nicht zur Geltung. Das gesamte Alkali darf daher unbedenklich von Anfang an zugesetzt werden. Damit sind die günstigsten Berlingungen für diese Reaktionen festgelegt.

Södertälje, Oktober 1921. 